$$
\text { PART I }
$$

\title{
ABSOLUTE MAGNITUDES \\ FROM TRIGONOMETRIC AND \\ STATISTICAL PARALLAXES
}




\title{
ABSOLUTE MAGNITUDES FROM TRIGONOMETRIC
}

\author{
AND STATISTICAL PARALLAXES
}

\author{
P. VAN DE KAMP \\ Sproul Observatory, Swarthmore College, Swarthmore, Pa., U.S.A.
}

\begin{abstract}
The attainable accuracy of photographic trigonometric parallaxes may be improved by the use of modern measuring engines, larger number of exposures, facilitated by increased speed of emulsions, more refined knowledge of reduction to absolute parallax. Systematic errors remain an obstacle both in trigonometric and statistical parallaxes.

With an attainable goal of \pm 0 !002 for the probable error of a parallax it appears difficult to obtain absolute magnitudes with a probable error less than 0.2 mag. for stars beyond $20 \mathrm{pc}$.
\end{abstract}

I believe that the best contribution I can make to the subject of absolute magnitudes is a presentation of the attainable accuracy in trigonometric parallaxes and a brief reference to the same problem in statistical parallaxes.

The evaluation of the accuracy of determining trigonometric annual parallaxes has been frequently studied and reported. Among the exhaustive studies, I mention those by Land (1948), Strand (1963), and Lippincott (1970). It is not easy to improve on these investigations but perhaps some general remarks may be in order today.

Greater accuracy may be reached in several ways. There is (1) the obvious choice of observing times, (2) the potential increase in accuracy due to the vastly increased speed of photographic emulsions, (3) the introduction of new measuring techniques, (4) better knowledge of reduction from relative to absolute parallax, (5) elimination of orbital motion in the case of series of observations covering a long time interval, both for known visual binaries and perturbations, (6) recognition and possible elimination of systematic errors, which vary with the interval of observation, (7) as far as absolute magnitudes are concerned: recognition of duplicity in order to obtain proper absolute magnitudes of the components.

\section{Observing Times}

Ideally, or rather traditionally, long focus photographic parallax observations are made at dawn and at dusk, for the purpose of obtaining the largest possible range in parallax factors in right ascension. The extreme range thus obtainable varies with the position of the star in the sky, the observer's latitude, and the pattern of clear nights as it varies with the time of the year. A large range in parallax factors, up to a maximum of 2.0, may be obtained for stars in certain right ascensions, while in other parts of the sky, at best, one has to be satisfied with smaller range, say only 1.5 , and this may not even be possible if weather conditions are unfavorable. As a rule a smaller range in right ascension is accompanied by a larger range of parallax factors in declination. At the Sproul Observatory, as a matter of policy, all stars are measured in declination also, not primarily for parallax, but for the obvious purpose of observing orbital motion 
(or perturbation). While on the average the weight of parallax determinations in declination is only $15 \%$ of that in right ascension, this procedure statistically, at least, has contributed to an evaluation of systematic accuracy. From a study of 124 stars, Lippincott has found an average difference, in the sense R. A. minus Decl., of -0 ".0018 \pm 0 ".0011 (p.e.). While this is no proof that either or both coordinates are free from systematic errors, the close agreement may be taken to exclude large systematic errors, at least on the average, for the whole sky.

In the early days of long-focus photographic parallax determinations, measurements were frequently made in celestial longitude, thus yielding a somewhat larger range in parallax factors. I do not believe that a return to this approach should be made. In all observational work it remains important to measure in basic, natural coordinates, as they result from the unavoidable observational approach and to study the errors, both accidental and systematic, inherent in this approach. Right Ascension and Declination are these natural coordinates, affected in a straight forward manner by such errors as are due to tracking, or guiding, in right ascension, while refraction and dispersion play a primary role in declination. For observations off the meridian, refraction and dispersion enter into right ascension, proportional to the hour angle and with opposite signs, for eastern or western hour angles. Schlesinger and others pointed this out and argued for as small a range in hour angle as possible, preferably hovering around the meridian. Personally, I take a very dim view of certain recent experiments with extremely large ranges in hour angle. This, in my opinion, is asking for trouble, no matter how elaborate the system of parameters and computer facilities may be. I continue to believe that prevention is better than the cure.

Ideally, parallax factors (in right ascension) of +1.0 and -1.0 are the astrometrist's dream. However, as pointed out earlier this ideal usually remains a castle in the air. This is no reason for despair; after all, it is the range in parallax factor which counts.

There is the related problem of matching the number of evening and morning observations. While ideally the number of plates with positive and negative parallax factors should be evenly matched, in practice one may settle or have to settle for considerable imbalance without much harm. Assuming a distribution of material which permits elimination (i.e., determination) of proper motion, the weight of a parallax determination depends on the total weight of the material and the range in parallax factors. To illustrate this, let us assume equal negative (evening) parallax factors $(-P)$ and equal (morning) parallax factors $(+P)$, hence a range $2 P$ between morning and evening. Assume fractions $f$ and $1-f$ of the material with opposite parallax factors. The weight of the parallax determination is given by

$$
n P^{2}\left[1-(1-2 f)^{2}\right]=n P^{2}\left[4 f-4 f^{2}\right],
$$

where $n$ is the total weight of the material (see following table on next page).

An imbalance in the ratio 2 to 1 leads to a weight reduction by a factor 0.89 , a ratio 3 to 1 to a reduction of 0.75 . Such imbalance, if accompanied by a good range in parallax factors, may often be quite an acceptable compromise, if not the only obtainable goal. 


\begin{tabular}{llll}
\hline$f$ & $1-f$ & $\begin{array}{l}\text { Weight of } \\
\text { parallax }\end{array}$ & $\begin{array}{l}\text { Relative } \\
\text { weight }\end{array}$ \\
\hline & & & \\
0.5 & 0.5 & $n P^{2}$ & 1.00 \\
0.6 & 0.4 & $0.96 n P^{2}$ & 0.96 \\
0.7 & 0.3 & $0.84 n P^{2}$ & 0.84 \\
0.8 & 0.2 & $0.64 n P^{2}$ & 0.64 \\
0.9 & 0.1 & $0.36 n P^{2}$ & 0.36 \\
\hline
\end{tabular}

\section{Photographic Emulsion; Law of Diminishing Returns}

An important historical factor in obtaining higher accuracy is the tremendous increase in sensitivity of photographic emulsions, conservatively speaking, fifty fold over the past fifty years. Where 'in the old days' we used to struggle to get 20 plates for a parallax determination, with the same observational effort we can now obtain 200 plates or more. The resulting increase in accuracy of a parallax determination is obvious if we may assume that increase of the number of exposures yields a corresponding increase in accuracy, i.e., that the law of diminishing returns does not enter to an appreciable extent. There is some evidence for small inhibiting effects of this law in the form of night errors which discourage an unduly enlarged observational effort for any one star on any one night. I feel that 16 to 20 exposures distributed over four plates still appear a desirable maximum goal for any one night.

\section{Measurements on Modern Machines}

Measurements on modern machines have proven that an impersonal evaluation by photoelectric scanning of photographic star positions together with superior precision screws, such as in the Grant two-screw machine, or the sophisticated optical approach, which is the basis of the U.S. Naval Observatory machine, lead to a substantially improved positional accuracy. A good indication of the superior accuracy obtained with the USNO machine is a comparison of measurements made by John L. Hershey of plates on CC 1213 taken with the Sproul 24 in. refractor over the interval 1959 to 1969.

\begin{tabular}{lcl}
\hline Machine & No. of reference stars & P.E. 1 \\
\hline Manual Swarthmore & 3 & $\pm 0^{\mathrm{mm}} .00139$ \\
USNO & 3 & $\pm 0^{\mathrm{mm}} .00098$ \\
USNO & 8 & $\pm 0^{\mathrm{mm}} .00096$ \\
USNO & 11 & $\pm 0^{\mathrm{mm}} .00091$
\end{tabular}

Provisional results obtained with the 2 -screw Grant machine now in operation at the Sproul Observatory indicate a similar favorable factor of weight increase. We list the following comparison; the positions refer to 3 to 5 reference stars. 
P.E. 1

One-screw machines built in 1916, 1943, 1963

Grant 2-screw machine $\pm 0^{\mathrm{mm}} .0020$

$\pm 0^{\mathrm{mm}} .0012$

The decrease in probable error from the bisection to the scanning method is striking. If these results are confirmed and maintained we reach the important conclusion that automatic or semi-automatic positioning by modern measuring engines leads, conservatively speaking, to a doubling of weight compared with earlier manual bisections. Here then is a significant accomplishment toward increasing the accuracy of longfocus trigonometric parallaxes. It raises the question whether all existing early plate material should be measured, to double, in a manner of speaking, that material, or rather, for the first time to exploit this material to the fullest; to extract from it all that can be obtained in the way of positional accuracy.

I view with reservation certain current attempts to materially increase parallax accuracy by using a large number of reference stars. The weight of a position referred to $n$ reference stars is proportional $n /(n+1)$ for the ideal case of equal dependences, or weights, of the reference stars. The increase in weight with increasing number of reference stars is seen from the following tabulation:

\begin{tabular}{lll}
\hline $\begin{array}{l}\text { No. of reference } \\
\text { stars }\end{array}$ & Weight & $\begin{array}{l}\text { Weight relative to } \\
\text { 3 reference stars }\end{array}$ \\
\hline & & \\
3 & 0.75 & 1.00 \\
4 & 0.80 & 1.07 \\
5 & 0.833 & 1.11 \\
6 & 0.857 & 1.14 \\
7 & 0.875 & 1.17 \\
8 & 0.889 & 1.19 \\
- & - & - \\
11 & 0.917 & 1.22 \\
- & - & - \\
$\infty$ & 1.00 & 1.33 \\
\hline
\end{tabular}

Recent results obtained at Sproul for CC 1213 yield relative weights of 1.04 and 1.11 for 8 and 11 star-, relative to the 3 star-configuration, actually below the values 1.19 and 1.22, which hold for equal dependences; all this for the case of linear plate constants. Introduction of other parameters, such as magnitude, color and non-linear terms, may be a reason for using more reference stars. Experience will tell.

\section{Reduction from Relative to Absolute Parallax}

This correction did not have to be taken too seriously in the days of conventional 
parallax determinations based on some twenty plates and yielding probable errors of about \pm 0 ".010. But currently, when such determinations are sometimes based on hundreds, or even thousands of plates, and with the vastly improved measuring techniques, probable errors of \pm 0 ".003 and less are becoming commonplace. Where in the past statistical corrections, based on magnitude and galactic latitude were adequate, there is now need for refining the evaluations of the parallaxes of the reference stars. In my opinion magnitude, spectra, coupled with some knowledge of the proper motions of the reference stars, are the best parameters for estimating the parallaxes of the reference stars and, hence, the reduction to absolute in the form of the dependence weighted mean of their individual parallaxes. An increase in number of reference stars has been proposed as a means of improving the reduction to absolute. In principle, this is true; in practice, little improvement would be reached.

\section{Elimination of Orbital Motion (Perturbation)}

Early parallax determinations based on a series of plates extending over a few years only, could hardly be affected by orbital motion. Series spread over several decades are needed in the determination of mass-ratio and perturbations. The conditional equation for proper motion and (relative) parallax:

$$
X=c+\mu t+\pi P
$$

is now replaced by

$$
X=c+\mu t+\pi P+\alpha Q
$$

or its equivalent, where $\alpha$ is the semi-major axis of the orbit of the observed position (primary, secondary, or photocenter). In case of known or recognized orbital motion, allowance can be made and the parallax determination will thus be not affected by orbital motion. It is likely however that for a number of-alleged-single stars orbital motion may go undetected and thus affect the parallax depending on the relative size of $\pi$ and $\alpha$.

\section{Recognition of Systematic Errors Depending on the Interval of Observation}

While the afore mentioned orbital effects would be different for different stars, there also may be systematic effects depending on the time interval which have nothing to do with the possible orbital behaviour of the individual star, but which depend on the specific time interval, decade $1960-70$ for example, and/or on the right ascension and declination of the star.

There is evidence for such effects in the recent study by Miss Lippincott (1970).

These effects may be related to adjustments of the optics of the telescope, or possible subtle changes in observing procedures. 


\section{Recognition of Duplicity in Order to Obtain Proper Absolute Magnitudes}

The attainable accuracy in parallaxes has some bearing on the determination and interpretation of absolute magnitudes. Is the observed star single or an unrecognized binary, not detected by any other means? An abnormally bright absolute magnitude may often be regarded with reservation or suspicion, and may be indicative of duplicity.

All in all, we may look forward toward appreciably improved accuracy of trigonometric parallaxes. Increases in weights up to a factor of 10 or more are obtained, or within reach, for large numbers of stars. The corresponding decrease in probable error, therefore, permits us with the same accuracy as before to explore stellar distances by the long-focus photographic method at least three times farther than we have done so far. But there are still other problems. Increased accidental accuracy does not at all necessarily mean increased systematic accuracy. The bug bear of systematic errors remains with us, although it may eventually become easier to pinpoint these errors because of increased accidental accuracy.

A similar pessimistic attitude may be maintained toward such ambitious projects as determining the trigonometric parallax of a star cluster such as the Hyades. The parallax of the Hyades is close to +0.025 and is rather well known from studies of the perspective effect in the proper motions of the cluster members. To improve this value we should reach a probable error of well below \pm 0 ".001 for the trigonometric parallax of the cluster. This expected value, would be based on many determinations for individual members of the cluster. With an investment of several thousand plates such a small error may well be reached, formally speaking, but who is going to assess the systematic error of such a result?

A few final remarks concerning the use of parallaxes. The principal significance of determining trigonometric parallaxes is their use for determining absolute magnitudes $(M)$ and masses $(\mathscr{M})$ :

$$
\begin{aligned}
& M=m+5+5 \log p \text { or } \log L=0.4\left(m-M_{\odot}\right)-2-2 \log p \\
& \mathscr{M}_{1}+\mathscr{M}_{2}=\frac{a^{3}}{P^{2}} \cdot \frac{1}{p^{3}} \text { or } \log \left(\mathscr{M}_{1}+\mathscr{M}_{2}\right)=\log \frac{a^{3}}{P^{2}}-3 \log p .
\end{aligned}
$$

The error situation is as follows:

$$
\begin{aligned}
& \mathrm{d} M=2.2 \frac{\mathrm{d} p}{p} \quad \frac{\mathrm{d} L}{L}=-2 \frac{\mathrm{d} p}{p} \\
& \frac{\mathrm{d}\left(\mathscr{M}_{1}+\mathscr{M}_{2}\right)}{\mathscr{M}_{1}+\mathscr{M}_{2}}=-3 \frac{\mathrm{d} p}{p} \quad \mathrm{~d} \log \left(\mathscr{M}_{1}+\mathscr{M}_{2}\right)=-1.3 \frac{\mathrm{d} p}{p}
\end{aligned}
$$

The fractional errors in the luminosity and in the total mass of a binary are proportional to the fractional error of the parallax; the same holds for the masses of the components, ignoring the error in the mass ratio. To maintain the same relative accuracy at greater distances, the weight of the parallax determination should increase with the square of the distance. We remind ourselves of the well known factor 3 , which 
makes it difficult to obtain precise masses even for the nearest visual binaries.

The following table summarizes the ratios between the fractional errors of the various quantities and the fractional error in parallax:

$\begin{array}{lll}\text { Absolute Magnitude }+2.2 & \text { Luminosity }-2 \\ \text { Mass } & -3 & \log \text { Mass }-1.3 \\ \frac{\text { Absolute Magnitude }}{\log \text { Mass }}-\frac{5}{3} & \\ \begin{array}{ll}\text { Luminosity } \\ \text { Mass }\end{array} \quad+\frac{2}{3} & \\ \quad \text { Luminosity } & -0.9 \\ \text { Absolute Magnitude } & \\ \frac{\text { Mass }}{\log \text { Mass }}+2.3\end{array}$

An example of the error situation is given below:

\begin{tabular}{lrll}
\hline Parallax & \multicolumn{1}{l}{$\begin{array}{l}\text { Probable } \\
\text { Error }\end{array}$} & $\begin{array}{l}\text { Probable Error } \\
\text { in abs. mag. }\end{array}$ & $\begin{array}{l}\text { Fractional Error } \\
\text { in total mass }\end{array}$ \\
\hline 0 & & & \\
0.100 & \pm 0.005 & 0.11 & 0.15 \\
& 0.002 & 0.04 & 0.06 \\
0.050 & 0.001 & 0.02 & 0.03 \\
& \pm 0.005 & 0.22 & 0.30 \\
0.020 & 0.002 & 0.09 & 0.12 \\
& 0.001 & 0.04 & 0.06 \\
& \pm 0.005 & 0.5 & 0.75 \\
& 0.002 & 0.2 & 0.30 \\
& 0.001 & 0.1 & 0.15
\end{tabular}

The tabulated figures speak for themselves. Assuming a probable error of \pm 0 "002 for parallax as a reasonable and obtainable goal, it becomes difficult to obtain stellar masses with a relative probable error smaller than $10 \%$ for stars beyond $20 \mathrm{pc}$. As to absolute magnitudes it appears difficult to obtain them with a probable error less than $0.2 \mathrm{mag}$. beyond $20 \mathrm{pc}$. And we have not yet taken into account, since we cannot, systematic errors!

On the subject of absolute magnitudes from statistical parallaxes, my colleague Luyten will deal with this problem particularly as it refers to the fainter end of the luminosity curve.

I have not been active in this field for several decades and feel that I cannot do much more than refer to the following survey papers:

(1) 'Statistical Parallaxes', by A. N. Vyssotsky, The Astronomical Journal 63, 166, presented at the Conference on the Cosmic Distance Scale, held in Charlottesville, Virginia, April 5-7, 1956. 
Vyssotsky stresses the limitations of the two principal methods used for deriving mean parallaxes: parallactic motions and dispersion in peculiar motions, i.e. $v$ - and $\tau$-components.

As to parallactic motions, they are sensitive to systematic errors in proper motions and errors in the precession constants. Moreover, knowledge of radial velocities is required.

As to peculiar motions, high accuracy is required as well as knowledge of radial velocities.

An additional complication is, of course, the dispersion in absolute magnitudes which affects the conversion from mean parallax to mean distance.

(2) 'Statistical Parallaxes', by Fricke (1964), presented at Aspects of Stellar Evolution, Flagstaff, Arizona, June 22-24, 1964.

Fricke points out the existence of errors up to 0.2 and more depending on the fundamental system used.

\section{References}

Fricke, W.: 1964, Vistas in Astronomy 8, 211.

Land, G.: 1948, Trans. Astron. Obs., Yale University, Vol. 15, Part 3.

Lippincott, S. L.: 1970, Publ. Leander McCormick Obs. 16, 99.

Strand, K. Aa.: 1963, Stars and Stellar Systems 3, 55.

Vyssotsky, A. N.: 1956, Astron. J. 63, 166. 